\title{
Reaction tubes: a new platform for silicon nanophotonic ring resonator sensors
}

\author{
C. Lerma Arce, ${ }^{1,4, a)}$ S. Van Put, ${ }^{2}$ A. Goes, ${ }^{3,4}$ E. Hallynck, ${ }^{1,4}$, P. Dubruel, ${ }^{3,4}$ K. \\ Komorowska, ${ }^{1,4}$ and P. Bienstman ${ }^{1,4}$ \\ ${ }^{1}$ Photonics Research Group (INTEC) Gent University, Sint-Pietersnieuwstraat 41, 9000 Gent, Belgium \\ ${ }^{2}$ Center for MicroSystems Technology, Gent University, Technologiepark 914, 9052 Zwijnaarde (Gent), Belgium \\ ${ }^{3}$ Polymer Chemistry and Biomaterials Group, Gent University, Krijgslaan 281 (S4), 9000 Gent, Belgium \\ ${ }^{4}$ Center for Nano- and Biophotonics, Gent University, 9000 Gent, Belgium.
}

Label-free biosensing with silicon nanophotonic ring resonator sensors has proven to be an excellent sensing technique for achieving high throughput and high sensitivity, comparing favorably with other labeled and label-free sensing techniques. However, as in any biosensing platform, silicon nanophotonic ring resonator sensors require a fluidic component which allows the continuous delivery of the sample to the sensor surface. This is the big disadvantage of this platform since this type of microfluidic system is very much removed from the daily practice in e.g. hospital labs, which still relies to a large degree on platforms like 96-well microtiter plates, or reaction tubes. To address this major drawback, we propose the combination of a simple and lab-compatible reaction tube platform, with label-free nanophotonic biosensors with a special microfluidic system imbedded into the same chip, where the flow is through the chip rather than over the chip as in more traditional approaches. This shows that label-free nanophotonic ring resonators can be also used in the user-friendly platform like reaction tubes or well microtiter plates, conserving their excellent performance.

\section{INTRODUCTION: THE MANUSCRIPT}

Millions of reaction tubes, as well as 96-well microtiter plates, are used every day in hospitals and laboratories to perform bioassays which serve as a diagnostic tool in medicine and plant pathology, as well as a quality-control check in various industries.

Those handy and disposable platforms for small fluid volumes have received large investments to achieve automatic and efficient handling optimizing the bioassays' performance.

However, these platforms only allow for labeled detection techniques which are labour intensive and costly and can structurally and functionally alter the assay. Quantification is difficult since the label intensity level is dependent on all working conditions. Moreover a labeled assay can only be performed in an 'end-point' fashion so that no kinetic information on the biomolecular interaction can be obtained. Also, label-based assays require a high degree of development to assure that

\footnotetext{
a) Cristina Lerma Arce. Electronic mail: Cristina.Lermaarce@ Intec.ugent.be.
} 
the label does not block an important active site on the tagged molecule or modify the molecular conformation.

Due to these considerations, there has been a drive to reduce assay cost and complexity while providing more quantitative information at high throughput [1]. Label-free detection is a solution to this involving a transducer that directly measures some physical property of the biological compound. This transducer can be mechanical [2,3], electrical $[4,5]$, or optical $[6,7]$.

Optical label-free biosensors have received considerable attention over the past years [6-9]. The key behind optical biosensors' ability to detect biological analytes is that they are able to translate a change in the propagation speed of light into a quantifiable signal proportional to the amount of biological material present on the sensor surface.

The type of biosensor considered in this work is an affinity-based biosensor: a so-called 'receptor' or 'ligand' is attached to the surface of the sensor, which responds to the affinity interaction of the receptor with an analyte. The receptor molecule can be an antibody, receptor protein or DNA. The formation of complexes can thus be monitored continuously and many interactions can be followed simultaneously. This real-time data results in information on the kinetics of the reaction as well as on the concentration of the antibodies in the sample.

Here we show that photonic ring resonators can also be used in these convenient and disposable platforms like reaction tubes or well microtiter plates, conserving their excellence performance as label-free biosensor.

The rest of this paper is structured as follows. Section 2 provides a description of the photonic chip layout as well as the embedded microfluidic system and concludes with the integration of reaction tubes and photonic sensors. Section 3 presents the proof-of-principle experiment performed describing in detail the setup required and analyzing the results of such experiment. Conclusions will be given in Section 4.

\section{INTEGRATION OF CHIP, MICROFLUIDICS AND REACTION TUBE}

\section{A. Chip layout and setup}

The photonic chip was fabricated in silicon-on-insulator (SOI) with 2- $\mu \mathrm{m}$ buried oxide and a 220 -nm silicon top layer using CMOS compatible 193-nm optical lithography and dry etching [10]. The ring resonators consist of 450-nm-wide single-mode waveguides, with 5- $\mu \mathrm{m}$ bend radius, $2-\mu \mathrm{m}$-long directional couplers, and a gap of $180 \mathrm{~nm}$ between the waveguides.

The rings support modes that resonate at a wavelength

$$
\lambda_{\text {resonance }}=\frac{L \cdot n_{\text {eff }}}{m}
$$

where $\mathrm{L}$ is the round trip length, $\mathrm{n}_{\mathrm{eff}}$ the effective refractive index of the resonant mode and $\mathrm{m}$ its order $(=1,2 \ldots)$. The resonance results in a sharp dip in transmission. When molecular interaction takes place in the vicinity of the ring the 
refractive index changes locally, which results in a change of the effective refractive index of the resonant mode, and hence in a resonance wavelength shift which can be monitored by scanning the wavelength.

The chip layout is illustrated in Figure 1. Four rings are connected to one common input waveguide, each of them having a dedicated drop signal port. Five of these four ring series are placed independently next to another. The five input waveguides are simultaneously addressed through vertical grating couplers with a 2-mm-wide collimated beam from a tunable laser source TSL-510 from Santec Corporation. The output signals of the ring resonators are near-vertically coupled to free space by means of integrated grating couplers and are imaged with an infrared camera.

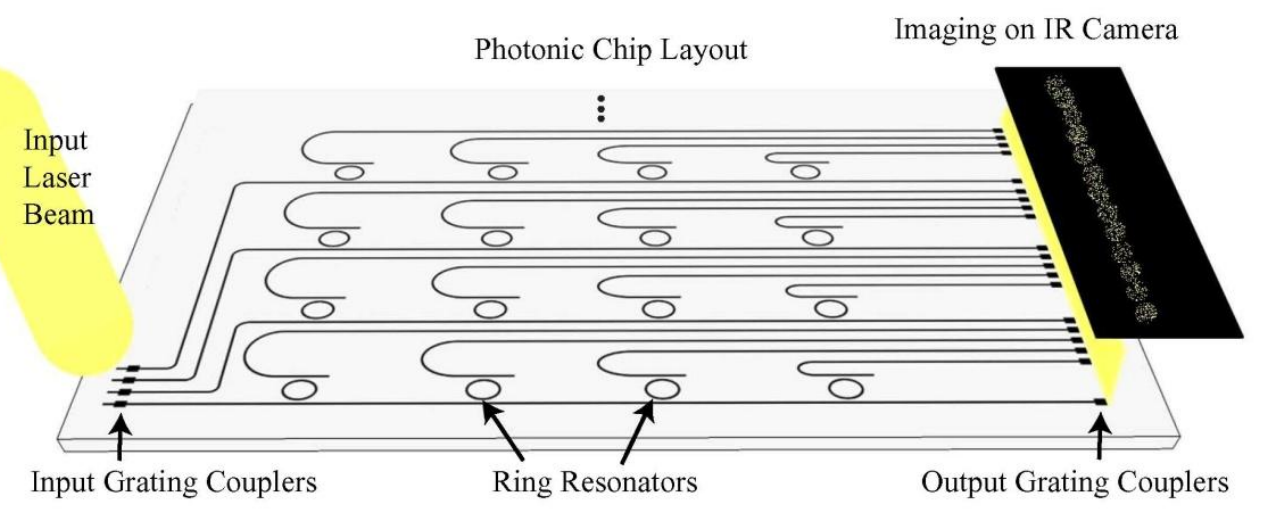

FIG. 1. Chip layout top view. A SANTEC TSL-510 tunable laser was used as a light source. The input waveguides are simultaneously addressed through vertical grating couplers with a $2 \mathrm{~mm}$-wide collimated beam from a tunable laser source. The output signals of the ring resonators are near-vertically coupled to free space by means of integrated grating couplers and are imaged with an infrared camera. This layout can be extended with four extra ring resonators for each extra input added.

The shallow-etched gratings are part of the chip design and have a maximum coupling efficiency of $31 \%$ at a wavelength of $1.55 \mu \mathrm{m}\left(40-\mathrm{nm} 1-\mathrm{dB}\right.$ bandwidth) for a $10^{\circ}$ off-vertical coupling angle [11]. Since the bandwidth of the grating couplers is larger than the free spectral range of the resonators, the grating couplers do not limit the number of resonators placed in series

\section{B. Integration}

The photonic chip is incorporated at the bottom of the reaction tube, once its original bottom is mechanically removed. The attachment of the chip to the bottom of the tube is done permanently using a UV curable adhesive which allows us to precisely align the array of sensors in the center of the reaction tube. Figure 2 shows a picture of the final device. 


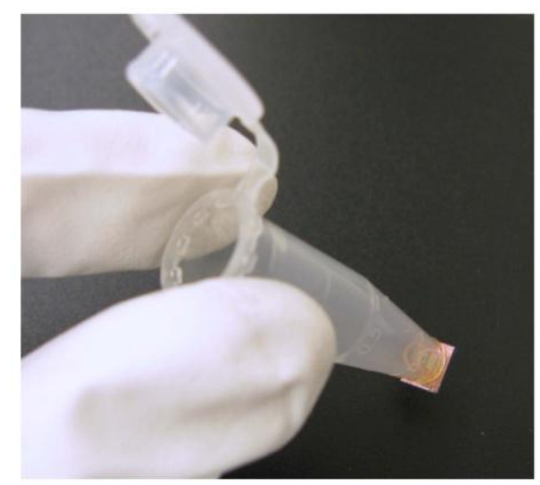

FIG. 2. The photonic chip is attached at the bottom of the reaction tube using a UV curable adhesive.

\section{Embedded microfluidic system}

Our purpose is to create a flow across the chip surface where the sensors are placed so the analyte to be detected is able to reach the vicinity of the sensor in a fast way.

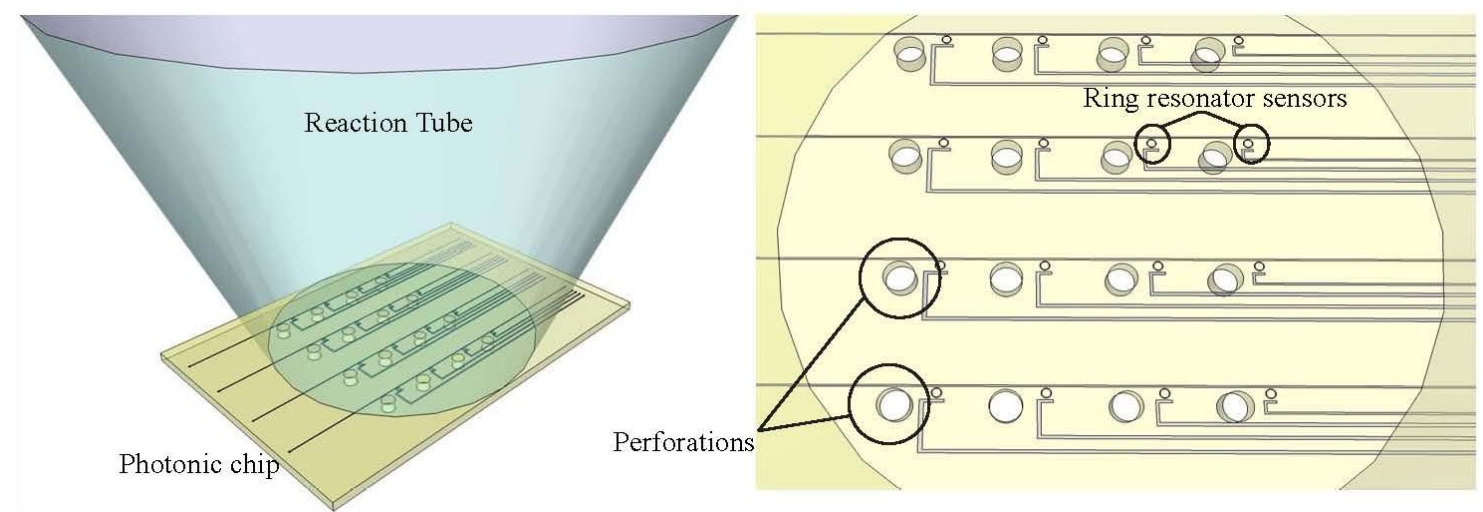

FIG. 3. a) Schematic of the assembled device. The silicon-on-insulator chip is incorporated at the bottom of a reaction tube. b) Layout of the chip. The array of ring resonators sensors is accompanied by an array of perforations next to them.

The flow within the tube is guided through certain apertures that perforate the chip. The solution inserted in the tube will flow through these openings which work as exit channels, creating the flow which will accelerate the detection process. Figure 3 shows a schematic of the device with the embedded microfluidic system.

The perforations through the silicon-on-insulator chip were laser ablated using a Duetto pulsed laser source from TimeBandwidth Products AG, operating at $355 \mathrm{~nm}$ with 1000 ps pulses with a repetition rate of $50 \mathrm{kHz}$. These perforations were done from the top to the bottom of the chip and its size and position can be easily optimized by customizing laser parameters. Figure 4 shows an array of photonic sensors accompanied by an array of perforations next to them. Each perforation will create a flow in its closest sensor. 


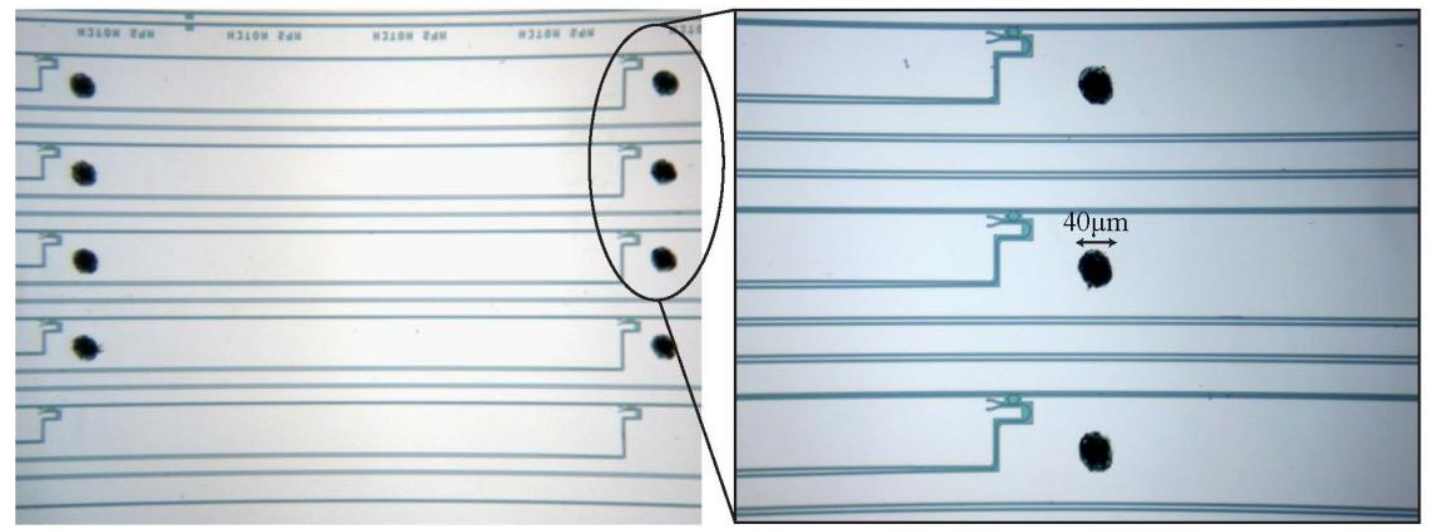

FIG. 4. Microscope picture of the perforations configuration. Each perforation will create a flow in its closest sensor.

In order to study the conditions of the flow in our system, we performed some basic 2D simulations in COMSOL.

We considered a system where an aqueous fluid was driven through a circular channel by imposing a pressure difference between an input and output. In this channel the volumetric flow rate is defined by the Hagen-Poiseuille equation [12]:

$$
Q=\frac{\pi r^{4}}{8 \mu} \cdot \frac{d P}{d z}
$$

where $r$ the radius of the cross section, $\mu$ is the dynamic viscosity of the fluid and $\mathrm{dP}$ the pressure drop along the length $\mathrm{dz}$ of the perforation through the chip. The mean velocity of the flow can then be calculated by:

$$
V_{\text {mean }}=\frac{Q}{A}=\frac{r^{2}}{8 \mu} \cdot \frac{d P}{d z}
$$

where $\mathrm{A}$ is the area of the cross-section of the channel.

We assume now that we have the geometry illustrated in Figure 5 .A water-based fluid is introduced in the first part of this geometry (i.e. the reaction tube), with a null-velocity, while a negative pressure is applied at the bottom of the geometry, creating an underpressure that drives the fluid through the different apertures (i.e. the perforations through the SOI chip) towards the exit of the channel.

Considering that the fluid introduced in the system is water, with a dynamic viscosity $1.002 \mathrm{mPa}$, the maximum velocity of the flow in the perforations was calculated to be $0.5 \mathrm{~m} / \mathrm{s}$ when applying a $1 \mathrm{kPa}$ pressure difference, which was confirmed by the simulations performed in COMSOL. For the geometry of our samples, and considering that the fluid introduced in the tube is water this velocity was determined to be $0.5 \mathrm{~m} / \mathrm{s}$ when applying $1 \mathrm{kPa}$ in one perforation. These calculations were confirmed by our model in COMSOL which was considered then as a reference. 


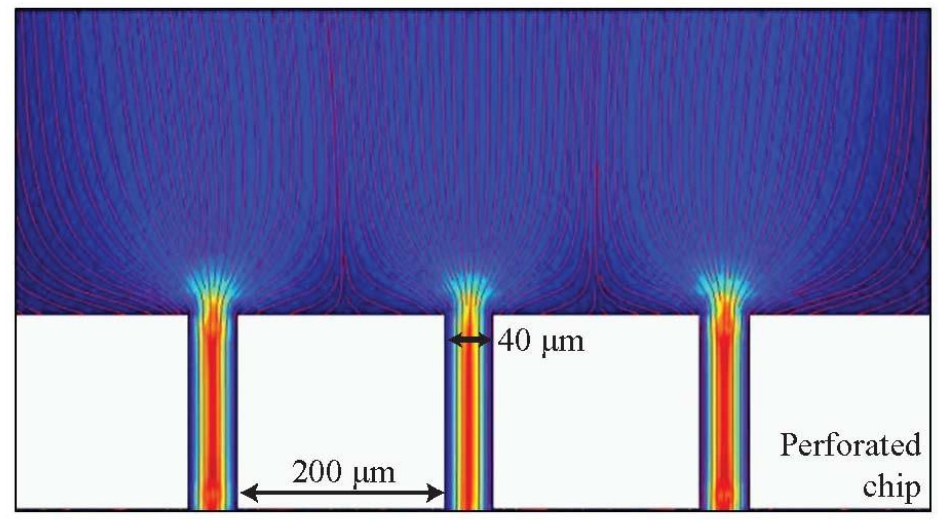

FIG. 5. Simulation of a water-based fluid flow in our device configuration. Streamlines in red confirm us the existence of a flow across the apertures and its vicinity.

The pressure applied on the system not only generates the transport of the fluid from the reaction tube to the exit. When looking in detail into the streamlines in the surroundings of one aperture (Figure 6) we discover the presence of vortices. Vortices are a natural effect of turbulent flows that can be also provoked in laminar flows. Normally, in laminar flows the mixing between different species relies heavily on mass diffusion, while when the vortices are generated the mixing performance is enhanced by convection. During the last two decades different methods for the fabrication of micromixers have been reported $[13,14]$ among which vortex generators stand [15]. We can say that the geometry of our model together with the pressure applied to the system stimulates the generation of vortices in the surroundings of the apertures which confirms that the transportation of the analyte to the surface of our sensors is done through convection. These vortices are stimulated every time that pressure is applied at the bottom of the system, making sure that the fluid on the chip is in continuous movement and delivering the analyte in an efficient way.

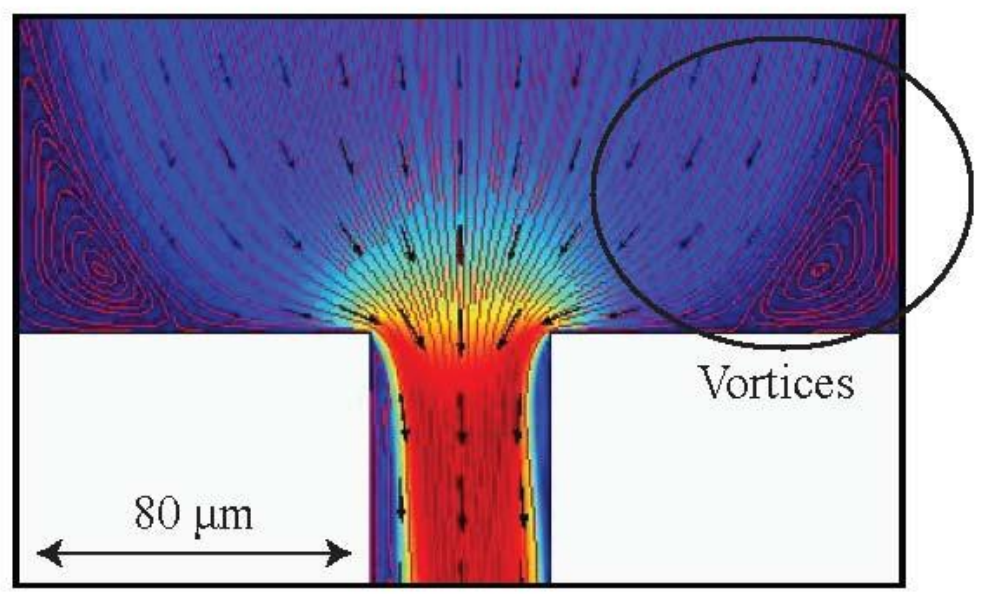

FIG. 6. Simulation detail of one of the apertures. Streamlines in red confirm us the existence of vorticity flow near the aperture. 
In conclusion, the perforations made through the SOI chip establish a flow through the sensors' surface. Depending on the position of these perforations the analyte will be brought to the sensor's surface by the main stream, which goes directly to the perforation and its very close vicinity, or by the vortices, a bit further from these perforations. In any of both cases the analyte will be transported in a fast and efficient way through a combination of convection and diffusion

\section{EXPERIMENTS}

\section{A. Setup}

To perform the experiments, the device was fixed on a tiny vacuum chuck that also has a connection to a pump, where pressure can be applied positively or negatively. This allows for pushing or sucking any gas of fluid applied in a specific area of the chuck. The reaction tube with the photonic chip integrated at its bottom was carefully aligned, so the perforations of the chip coincide with this area. Any fluid in contact with the chip will flow through the holes and be sucked down or pushed up again by the pump. Figure 7 shows the device fixed on this chuck.

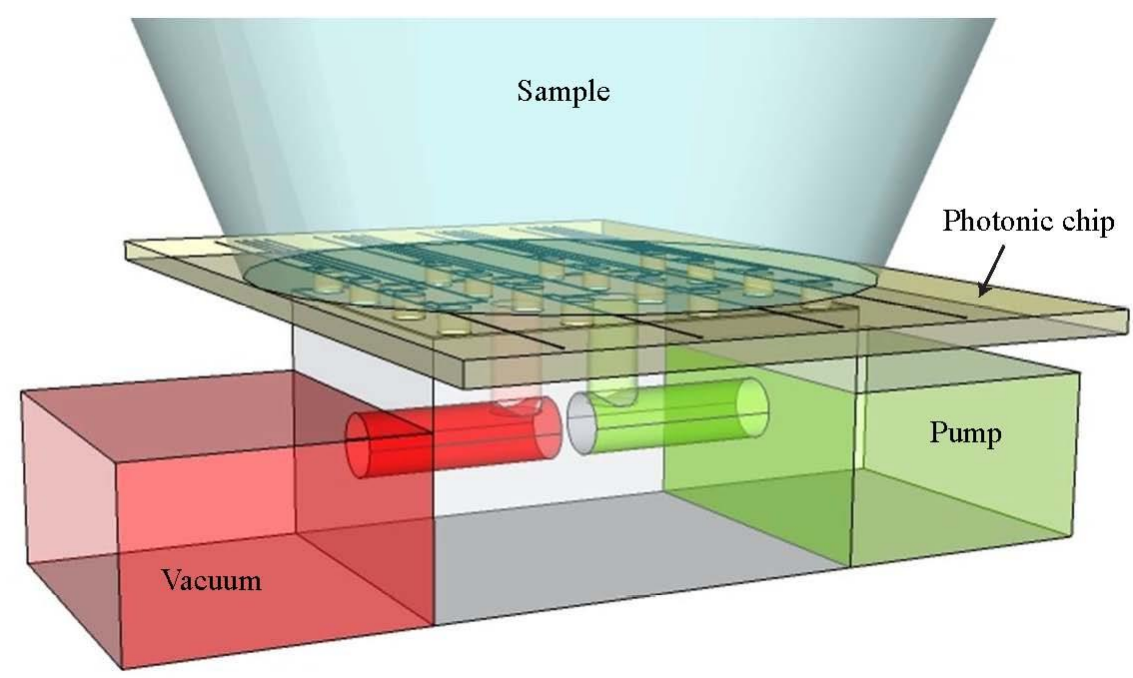

FIG. 7. Device mounted on a vacuum chuck with an additional pump to move the fluid across the chip surface.

As described in Section 2.1, grating couplers were used to couple the light from a tunable laser into the chip and couple it out again to be detected by an infrared camera. A new aspect with respect to our previous work [8] is that now, we couple light in and out from the bottom of the chip through the silicon substrate, as shown in Figure 8. 


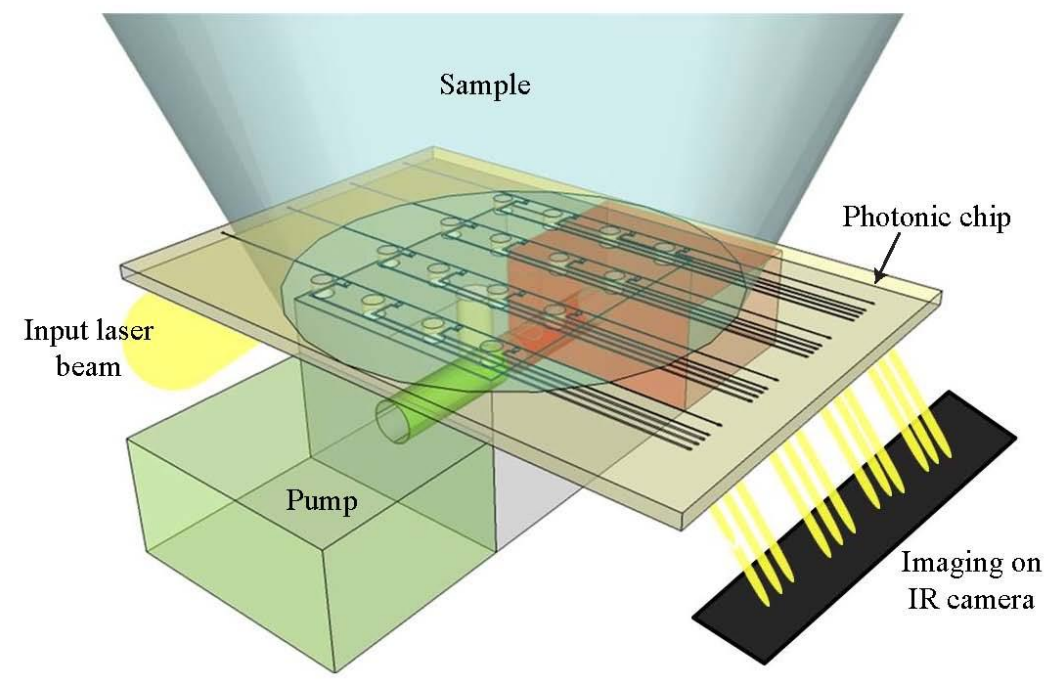

FIG. 8. Device mounted on a vacuum chuck, while measurements are being performed from the bottom of the chip. Light is coupled in and out from underneath, through the chip substrate.

Silicon features high transparency at the wavelength used $(1.55 \mu \mathrm{m})$. However, to reduce the scattering from the rough substrate surface and to facilitate both the alignment of the laser beam and the detection of the light coupled out from the chip, the silicon substrate was thinned down to $200 \mu \mathrm{m}$ and planarized by chemical-mechanical grinding (CMG) and polishing (CMP) in order to attain a smooth surface.

\section{B. Experimental results}

As a proof-of-principle and in order to show the capabilities of the combined device, we measured the response of the device when aqueous solutions with different sodium chloride concentrations were flown through the device. Small volumes of different solutions were manually pipetted into the tube, and they were sucked out through the perforations on the bottom of the tube while being measured. The change of refractive index in those solutions causes the shifts in wavelength observed in Figure 9. 


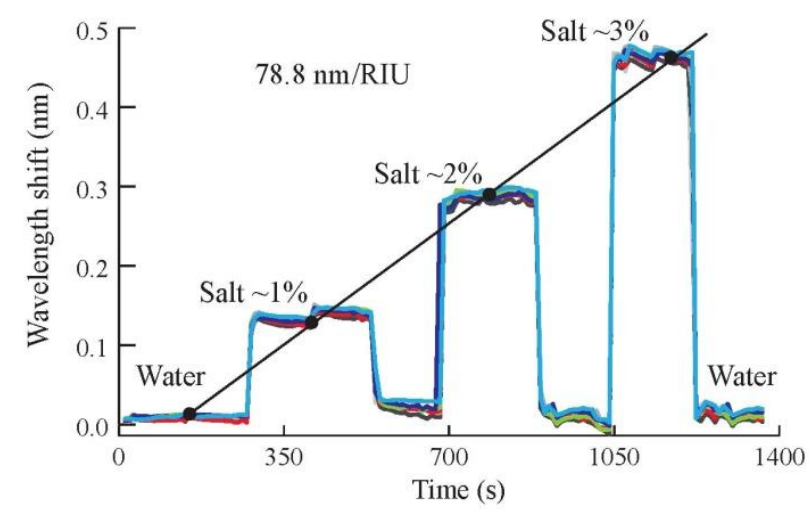

FIG. 9. Evolution in time of the resonance wavelength shift of the ring resonators during the measurements of different sodium chloride concentrations. Each color corresponds to one sensor. The shift of the resonance wavelength is easily measurable when changing from water to salt solution and vice versa. The sensitivity measured for these sensors is $78.8 \mathrm{~nm} / \mathrm{RIU}$, which confirms that the transducer does not suffer any degradation when being integrated into the device.

When analyzing these shifts as a function of the refractive index unit $\left(\mathrm{RIU}^{1}\right)$ the sensitivity measured for these biosensors is $78.8 \mathrm{~nm} / \mathrm{RIU}$ which corresponds once more with the measurements performed and proved in a our previous work [8], where these sensors where also used. This confirms that the transducer does not suffer any degradation when being integrated into the device.

Additionally, different steps of an assay where the well-known high-affinity couple biotin-streptavidin were measured. The actual surface chemistry used in this experiment was far from optimized, but this does in no way detract from the main point of this work, which is about showing the working principle of the new combined device. The experiment consisted of the following three individual steps:

1 Silanization of the surface: The surface was first modified with an amine-terminated silane by flowing $2 \%$ solution of 3-Aminopropyltriethoxysilane APTES in ethanol, both preceded and followed by rinsing with ethanol.

2 Immobilization of biotin: a solution $3 \mathrm{mg} / \mathrm{ml}$ of biotin in phosphate buffered saline (PBS) was flown, both preceded and followed by rinsing with PBS pH 7. This bound to the amine-terminated silane previously flown.

3 Binding of streptavidin: a solution of $0.1 \mathrm{mg} / \mathrm{ml}$ strepavidin in PBS was flown, both preceded and followed by rinsing with PBS pH 7. This bound to the biotin previously bound.

\footnotetext{
1 'Refractive index unit (RIU)' makes reference to the unit change of the refractive index of a measured fluid.
} 
Here again, all the volumes were manually pipetted into the tube, and they were sucked out through the perforations at the bottom of the tube while being measured. The time to perform each of these steps was less than one hour.

Figure 10 shows three different graphs corresponding to each one of the steps of the assay. They show the evolution in time of the resonance wavelength shift of the ring resonators during the measurements with different solutions. Each color corresponds to one sensor.

The association and disassociation of APTES in the sensor is easily quantifiable in Figure 10a. Figure 10b shows the binding of biotin after flowing $3 \mathrm{mg} / \mathrm{ml}$ solution of biotin in PBS and its disassociation when it is rinsed with PBS. In these two graphs, some intermediate discrete bulk shifts can be observed during the flow of APTES and biotin, which were provoked by the injection of new solution in the reaction tubes. In Figure 10c a shift of 30pm is measured when streptavidin is flown through the chip indicating the binding of this to biotin.
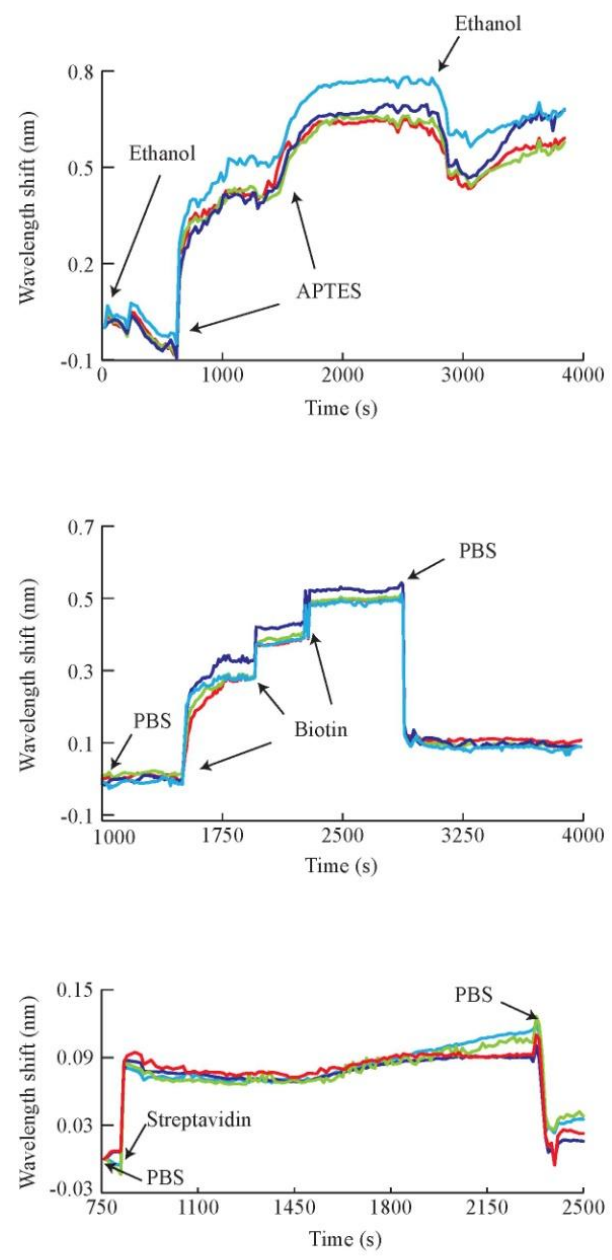

FIG. 10. Three different steps of the bioassay were measured: a) Silanization of the surface with APTES (ethanol - APTES - ethanol); b) Immobilization of the biotin (PBS - biotin - PBS); c) Binding of streptavidin to biotin (PBS - streptavidin - PBS) 


\section{CONCLUSIONS}

We have presented a new platform for silicon photonic ring resonators: reaction tubes.

We have combined the simplicity and portability of the reaction tube format with the performance and throughput of silicon photonic ring resonator biosensors by embodying them in a single device flow-through device.

An embedded microfluidic system based on perforations of the SOI chip attached to the tube provides an alternative to the typically costly and complex microfluidic system based on microchannels.

The disposability of the combined device is enhanced by the mass fabrication of SOI chips and the cheap and user friendly format of reaction tubes which are used every day in labs and hospitals, two factors that benefit their use for performing label-free bioassays.

We have proved no degradation of the sensors by bulk sensing experiments and the good performance of the device by showing the binding of streptavidin to avidin on the surface of the sensors. The device allows for multiplexed real-time detection and analysis. Its great flexibility, portability, and disposability make it ideal for easy and fast use in any laboratory.

\section{ACKNOWLEDGEMENTS}

We wish to thank our colleagues of the Center for MicroSystems Technology, the Polymer Chemistry and Biomaterials Group, and the Center for Nano- and Biophotonics, all at the Ghent University. We would also like to acknowledge ePIXfab (http://www.epixfab.eu) for the fabrication of the optical chips and Steven Verstuyft for fabrication support.

\section{REFERENCES}

${ }^{1}$ A. J. Qavi, A. L. Washburn , J. Byeon, R. C. Bailey, Anal. Bioanal. Chem., 394, 121 (2009)

${ }^{2}$ K. Länge, B. E. Rapp, and M. Rapp, Anal. and Bioanal. Chem., 391, 1509 (2008)

${ }^{3}$ A N. V. Lavrik, M. J. Sepaniak, and P. G. Datskos, Review of Scient. Instrum., 75, 2229, (2004)

${ }^{4}$ F. Patolsky and C. M. Lieber, Materials Today, 20 (2005)

${ }^{5}$ S. Schusser, M. Leinhos, M. Bäcker, A. Poghossian, P. Wagner, and M. J. Schöning, Physica Status Solidi A, 210, 905 (2013) 
${ }^{6}$ D.X. Xu, M. Vachon, A. Densmore, D. Zhang, R. Ma, Q. Y. Liu, A. Delâge, P. Cheben, S. Janz, J. Lapointe, J. H. Schmid, 35, 2771 (2010)

${ }^{7}$ M. Iqbal, M. A. Gleeson, B. Spaugh, F. Tybor, W. G. Gunn, M. Hochberg, T. Baehr-Jones, R. C. Bailey, L.C. Gunn, IEEE J. Quant. Electron., 16, 654 (2010)

${ }^{8}$ K. De Vos, J. Girones Molera, T. Claes, Y. De Koninck, S. Popelka, E. Schacht, R. Baets, P. Bienstman, IEEE Phot. J., 1, 225 (2009)

${ }^{9}$ C. E. Soteropulos, H. K. Hunt, A. M. Armani, Appl. Phy. Lett., 99, 103703 (2011)

${ }^{10}$ S. K. Selvaraja, P. Jaenen, W. Bogaerts, P. Dumon, D. Van Thourhout, R. Baets, J. Lightw. Technol., 27, 4076 (2009)

${ }^{11}$ D. Taillaert, P. Bienstman, R. Baets,, Opt. Lett., 29, 2749 (2004)

${ }^{12}$ Henrik Bruus. “Theoretical Microfluidics”. Oxford University Press, (2008)

${ }^{13}$ V. Hessel, H. Löwe, F. Schönfeld, Chem. Eng. Sci., 60, 2479, (2005)

${ }^{14}$ L. Capretto, W. Cheng, M.Hill, X. Zhang, Topics in Current Chemistry. Springer (2011)

${ }^{15}$ K.-Y. Hsiao, C.-Y.Wu, and Y.-T.Huang, Chem. Eng. Journal, 235, 27, (2013) 